\title{
Research of Image Stitching Method Based on Graph Cuts and Poisson Fusion
}

\author{
Yan Gong, Hong Xie, Wu Xie and Yun Lin \\ Information and Communication Engineering \\ Harbin Engineering University \\ Harbin 150000, China \\ gyan163@163.com
}

\begin{abstract}
This paper considers the problem of ghosting artifacts and stitching seam/caused by image stitching and fusion directly, which influenced by the registration and geometric transformation, and the differences between the two images overlap region. This paper proposed maximum flow / minimum cut in graph cut algorithm to obtain the optimal stitching line. Considering the relationship between color difference and structural changes of pixels in the neighborhood, achieving costs op energy division minimize, thereby eliminating the stitching seams. And using the Poisson fusiontechnology, further eliminate ghosting artifacts caused by the brightness and color differences ormoving objects in the image fusion process. The experiment proved that the algorithm of searching optimal stitching line and Poisson fusion algorithm in this paper, conleliminate ghosting artifacts and also be able to maximize avoid stitching seams appears, it presents goodperformance in applications.
\end{abstract}

Keywords: Image fusion, Stitching Mine graph cut, max flow / min cut, Poisson fusion technology

\section{Introduction}

Automatically-ereate a large) and high-resolution image stitching technology has been the active research field of photogrammetry, computer vision, image processing and computer graphics. In recent years, the rapid development of panoramic image stitching technology has been widely used in virtual reality, medical, aerospace, military and other fields. Therefore, it also put forward higher requirements on its core technology.

Panorama stitehing technology involves a lot of problems. For example, the image global / local alignment, local pixel adjustment of the alignment result image, the fusion pixel region selection etc. At present in the image acquisition, image captured by hand-held camera mostl, it often increase the difficulty of image stitching because of jitter. To solve the problem of geometric distortion and distortion stitching images also has an important significance. At the same time, the stitching of large exposure differences images often exist stitching marks. Thus, in recent years, image fusion technology more and more attention. How to eliminate the ghosting artifacts in the image stitching process, especially when the scene with moving objects, it is the difficulty of eliminating ghosting artifacts caused by moving object in image stitching field. In this paper, we propose use of finding the optimal stitching line to solve this problem. Because of the local optimal stitching line that is obtained by dynamic programming algorithm usually can not be a good segmentation of good stitching results, so this paper is based on the graph cuts algorithm to find the optimal stitching line, by min cut /max flow algorithm to achieve a minimum energy costs or split. At the same time, 
we use the Poisson image fusion algorithm to image fusion, in order to achieve the image fusion for color difference images and ghost removal.

\section{Searching the Optimal Stitching Line based on Graph Cut Algorithm}

Image stitching method based on the stitching line and pixel fusion have different ideas, the utility model has the advantages of the overlap region is divided into two blocks, a block reference image, the other taken pixels from the image will be stitching. That will not occur due to the emergence of fusion ghosting artifacts and aliasing.

We use the min cut /max flow algorithm based on the graph cuts algorithm to find the optimal stitching line.

\subsection{The Min Cut/Max Flow Algorithm}

Min cut / max flow algorithm is a classic algorithm of 20 mputer graph theory [1]. The methods include two categories, Push relabel method [2-3] and Augmenting paths method [4].

Push relabel method, along the edge of the unsaturated distance to the lower bound estimate of one to sink, then, facing the minimum estimated distance has to sink to advance the remaining vertex stream. With the push operation, the edgg gradually saturated, gradually increasing the distance.

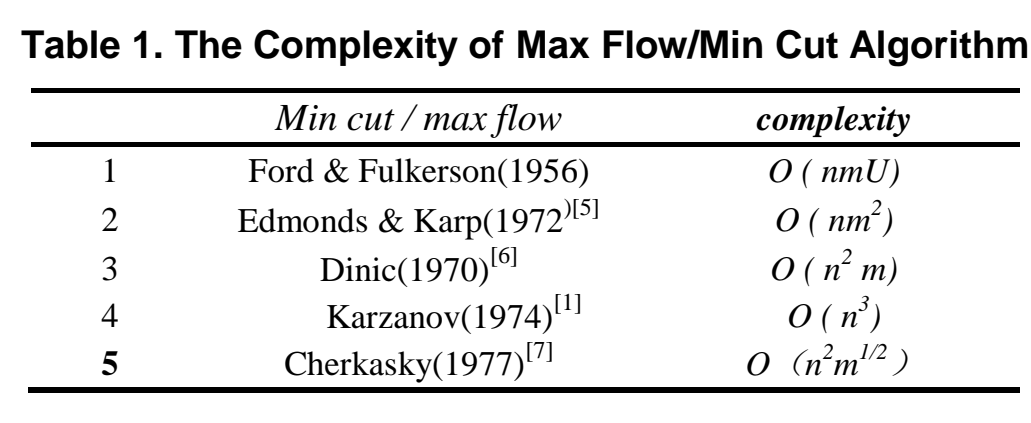

Relabel method of Ford and Fulkerson (FF for short) is a method of augmenting path based on, by labeling the continuous growth of a tree, until not to be finding the augmentation path of feasible flow sofary The computational complexity of the FF method is independent of the number of network nodes or edges, it depend on the weights of the edge. In order to avoid the maximum flow computation complexity, the weights depend on the edge of the defect, Dinic [6] design a hierarchical algorithm. In order to further improve the minimal cut / maximum floweffieiency, Boykov proposed a new method based on the augmented path, application of this method in the field of computer vision is the most widely, its core is to establish two search tree $S$ and $T$, the source point s as the root, $\mathrm{t}$ is the root to sink. All the edges of parent node to the child node in tree $S$ are not saturated. The nodes in the tree $S$ is divided to Active nodes and Passive nodes. Active node can make the search tree growth by obtaining new offspring from the tree $T$, the passive node cannot grow. The algorithm repeats the following three stages:

(1) Growth stage: The search tree $S$, T growth, until you find the meeting point.

(2) Augmentation stage: Expansion path, the search tree becomes forest.

(3) Adoption stage: The adoption of isolated node, restore the search tree.

As is shown in the figure 1, this is the completed state of two tree search tree finally. Active nodes marked with $A$ in the Figure 1, passive node marked with $P$, the free nodes are represented by the black circle. 


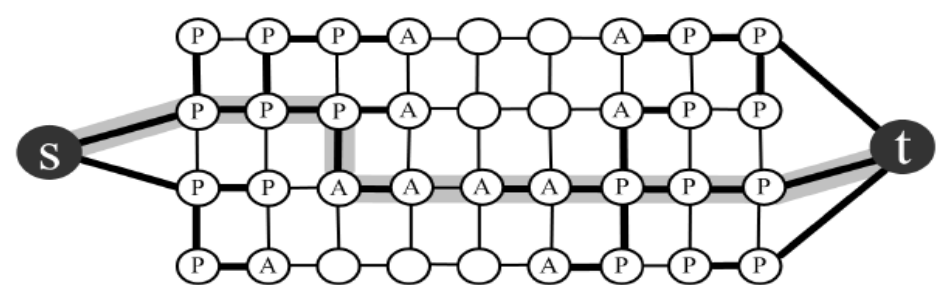

Figure 1. Searching Tree $S$ and $T$

The algorithm defines two no connecting search tree $\mathrm{S}$ and $\mathrm{T}$, respectively, growth ffom the source $s$ and sink point in $t$, the edges are not saturated. Therefore, we can get the conclusion:

$$
S \subset V, s \in S, T \subset V, t \in T, S \cap T=\Phi \mathcal{A}
$$

\subsection{Searching the Optimal Stitching Line}

In the image stitching system, the structure of the image and the graph can be expressed by the following chart [8].

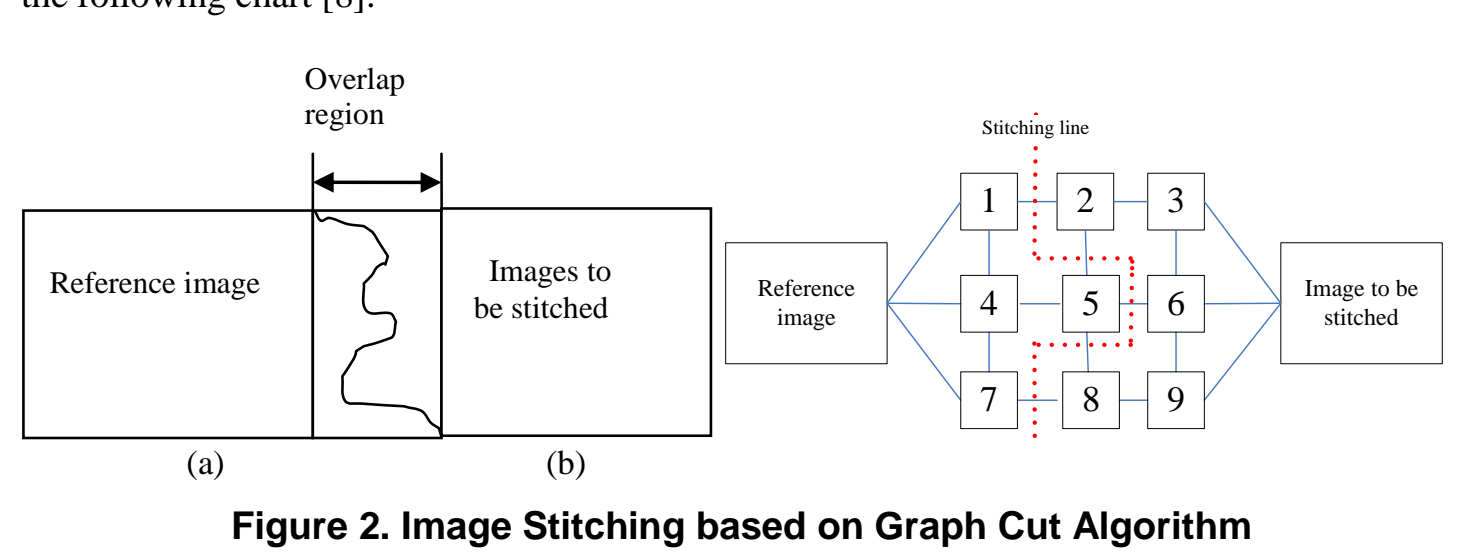

On the left is a schenatic diagram of image stitching, in which after the image to be stitched deformation, and the reference image with a certain overlap area, And the dividing line will be positioned in the overlap region, as shown by the red line is a sample line. Assuming overlap region of the reference image and the image to be stitched have only nine pixels, each pixel region as a node in the graph structure, then the nine squares from 1 to 9 to represent the nine adjacent nodes. There is a weighted line with a value between nodes, and the pixels in non overlap region, that is representation part of the "reference image" box and "images to be stitched" box in diagram, and nodes within the overlap region also has a weighted line with a value. The concept of the optimal stitching line search, which is in the overlap region, found a dividing line for the nodes in overlap region can be divided into two parts, one part from the reference image, the other part from the images to be stitched, this line from will across the nodes and cut weighted line between nodes directly.

According to this example, overlapping areas of the two large images can build a large graph structure. In constructing the graph, the most critical part is the definition of the weighted line. The definition of weighted line determines the form of energy function.

Calculation of the energy function is: 


$$
E(f)=\sum_{\{p, q\} \in N} V_{p, q}\left(f_{p}, f_{q}\right)+\sum_{p \in P} D_{p}\left(f_{p}\right)
$$

The energy function consists of the addition for data item and smoothness item. The search process of the stitching line is the energy function minimization process. We use max flow / min cut algorithm for the minimization of energy function.

In the initialization phase, we retain a result map, it's the final result will be our stitching results with the reference map to initialize it. Optional source which comprises a reference image and the image to be stitched, which has two sources points in the graph structure, the pixels can be derived from the reference image or image to be stitched in image stitching,

After initialization is complete, it enters the iterative minimization process of the algorithm. First, the energy function is calculated using the total energy of the carculation result. And optional source of $\alpha$-expansion, the definition of $\alpha$-expansion is:

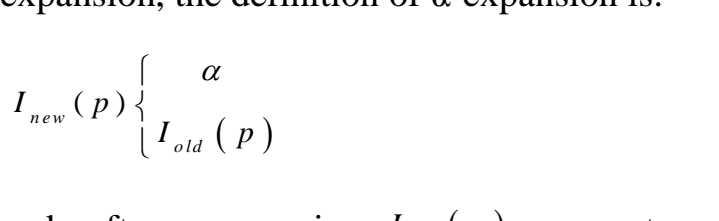

Which $I_{\text {new }}(p)$ represents $\mathrm{p}$ node after $\alpha$-expansion, $I_{\text {old }}(p)$ represent $\mathrm{p}$ node before $\alpha$ expansion, $\alpha$ represents corresponding p node jn an optionfabsource. Using the node in the optional source to replace the $\mathrm{P}$ node in results graph, then to calculate energy function of the current result graph, if the energy is reduced, it use alternative source node as a result of the nodes in the graph, or restore the grignal node. The whole algorithm iteration until the energy function is no longer reduced, so the final result image is the result of image stitching, which divided the optional source of the dividing qine is the optimal stitching line.

From the energy minimization process can be seen in the whole process to continuously calculate the energy function, and selecting according to the size of the energy function. It only gives the primary definition of the energy function above, and not related to specific forms of this function. We will define the calculation method of energy function, and discuss the difference between the various definition method and the stitching effect. The energy function consists of data thend smoothness item, the definition of a data item is a simple value as shown below

$$
D_{p}\left(f_{p}\right)=\left\{\begin{array}{cr}
0 & \text { if } p \in \text { over l ap } \\
+\infty & \text { else }
\end{array}\right.
$$

The definition of data items for this format in order to defined stitching line is located in the overlap region in the system, if the current node is not located in the overlap region, then the energy function value will be $+\infty$, it does not meet the requirements of energy minimization, so it's natural stitching line will be located in the overlap region. For smoothness item, it can through the CUT parameter selection to select different definitions way, the smoothness item of the system can use four kinds of definition, as listed below:

(1) Based on the color domain

$$
V_{p, q}\left(f_{p}, f_{q}\right)=\left\|f_{p}(p)-f_{q}(p)\right\|+\left\|f_{p}(q)-f_{q}(q)\right\|
$$

The definition of the smoothness item based on color domain considers only the pixel value of the pixel in image, and where $f_{p}$ and $f_{q}$ can be reference image or image to be stitched. Where $\|\bullet\|$ is the Euclidean distance defined by a RGB color values. 
(2) Based on the gradient domain

$$
V_{p, q}\left(f_{p}, f_{q}\right)=\left\|\nabla f_{p}(p)-\nabla f_{q}(p)\right\|+\left\|\nabla f_{p}(q)-\nabla f_{q}(q)\right\|
$$

The definition of the smoothness item based on gradient domain use the gradient value of pixels, including $x$ and $y$ directions of 6 RGB gradient component. And using Euclidean distance calculation, it uses the definition of smoothness item based on the gradients, can avoid overall difference of the colors domain that changes of illumination.

(3) color and gradient

$$
V_{p, q}\left(f_{p}, f_{q}\right)=V_{\text {colors }}+V_{\text {gradients }}
$$

We consider the effect of color and gradients at the same time, can bbtain more information of energy function.

(4) Based on image edge

$$
V_{p, q}\left(f_{p}, f_{q}\right)=\frac{V_{\text {colorp }}}{\left.G_{f_{p}}(p, q)+G_{p}, q\right)}
$$

The definition of smoothness item based on image edges đuses Sobel filter to extract edges, $G_{f_{p}}(p, q)$ and $G_{f_{q}}(p, q)$ use the Sobel filter to calculate below, including horizontal and vertical edge components $\mathrm{k}$ direction side component is calculated. Edge component to the horizontal direction and vertical diretion were calculated. The definition of smoothness item based on image edges can make the segmentation line as close as possible to the image, which makes the segmentation more difficult to detect.

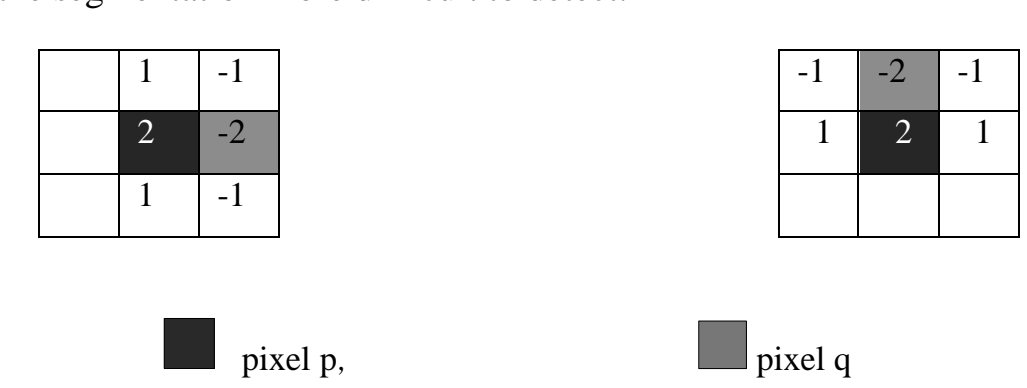

Figure 3. Sobel Filter

\section{Poisson Image Fusion Algorithm}

Aften the optimal stitching line search, still need to fusion synthesis image. Because the inevitable brightness difference between the source image and the image to be stitched, there will be the phenomenon of inconsistent luminance for target image, and stitching line near the moving objects, the overlap region and other reasons, will lead to the ghost artifacts.

\subsection{Ghosting Artifacts caused by Brightness Differences Removing}

Domain of the target image set $\mathrm{S}, \Omega$ is the area of the repair of $\mathrm{S}$, the boundary is

$$
\partial \Omega=\left\{p \in S \backslash \Omega: N_{p} \cap \Omega \neq \Phi\right\}
$$


Where $N p$ denotes 4-neighborhood of point $\mathrm{P}$. Suppose that $\mathrm{f}$ is the mixed color information defined on $\Omega \cdot f^{*}$ is the known color information on $\partial \Omega$. The process of solving mixed colors can be seen as interpolation on $\Omega$ which the known color of pixel on guide of repair color gradient field. It can be transformed into solving the following linear equations:

$$
\left|N_{p}\right| f_{p}-\sum_{q \in N_{p} \cap \cap_{\Omega}} f_{q}=\sum_{q \in N_{p} \cap \partial \Omega} f_{q}^{*}+\sum_{q \in N_{p} \cap_{\Omega}} v_{p q}
$$

Where, $p \in \Omega$ is the gradient of repair colors on repair area $\Omega v_{p q}=g_{p}-g_{q}, g_{p}$ and $g_{q}$ are repair color value on the repair area $\Omega$. Because it is a large sparse system of $\psi_{i n e a r}$ equations, we use conjugate gradient method to solve mixed color valua of REB three channels on repair area, it can quickly get mixed color value [9].

\subsection{Removing Ghost Artifacts caused by Moving Object}

After inputting the source image, we label all regions of objects to beremoved and create a gradient vector field by editing gradients of the source image. The object regions are removed from the source image and the gradient vector freld. In order to make the removed regions look like as if the image were captured without the objects in the scene, we recover them with the best-fit contents found in the other part of the image by the gradient domain region filling operation which is detailed in the next section. In this way, we can obtain a region filled composite image $I_{f}$ and a region filledomposite gradiènt vector field $(G x, G y)$.

The next step is to recover a hew composite rmage Ic from the gradient vector field $(G x, G y)$. In order to do this, we compute ardivergence vector field $\operatorname{div}(G)$ from the gradient vector field $(G x, G y)$ and use it as a guidance vector to construct a Poisson equation. The new composite image Ic can be recovered from the gradient vector field by solving the Poisson equation [10] with boundary condifions.

While solving the equation, we use image $I_{f}$ as a initial value, so that we can give the linear solver a good initial estimation of the solution.

Suppose $I(x, y)$ is our composite image. We use the divergence as a guidance to construct a Poisson equation,

$$
\nabla^{2} I(x, y)=\operatorname{div}(G)
$$

Where $\nabla^{2}$ is the Laplacian operator;

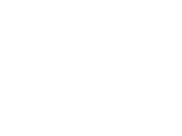

$$
\nabla^{2} I(x, y)=\frac{\partial^{2} I(x, y)}{\partial x^{2}}+\frac{\partial^{2} I(x, y)}{\partial y^{2}}
$$

$\operatorname{div}(G)$ is the divergence vector field;

$$
\operatorname{div}(G)=\frac{\partial G_{x}}{\partial x}+\frac{\partial G_{y}}{\partial y}
$$

This is a linear partial differential equation. In order to solve this equation, we must first specify boundary conditions. In our case, we use the Neumann boundary conditions.

Finally, the new composite image $I_{c}$ can be recovered from the gradient vector field $(G x, G y)$ by solving the Poisson equation. We use the result as our final composite image 
which can be used in the panorama stitching process for producing deghosted panoramic images.

\section{Applications and Result Analysis}

To verify the image stitching process proposed in this paper that uses the max flow / min cut method for obtaining the optimal stitching line, and the brightness, color differences of images fusion based on Poisson image fusion algorithm and ghost artifacts removed, we select 6 common river side pictures to stitching and fusion.

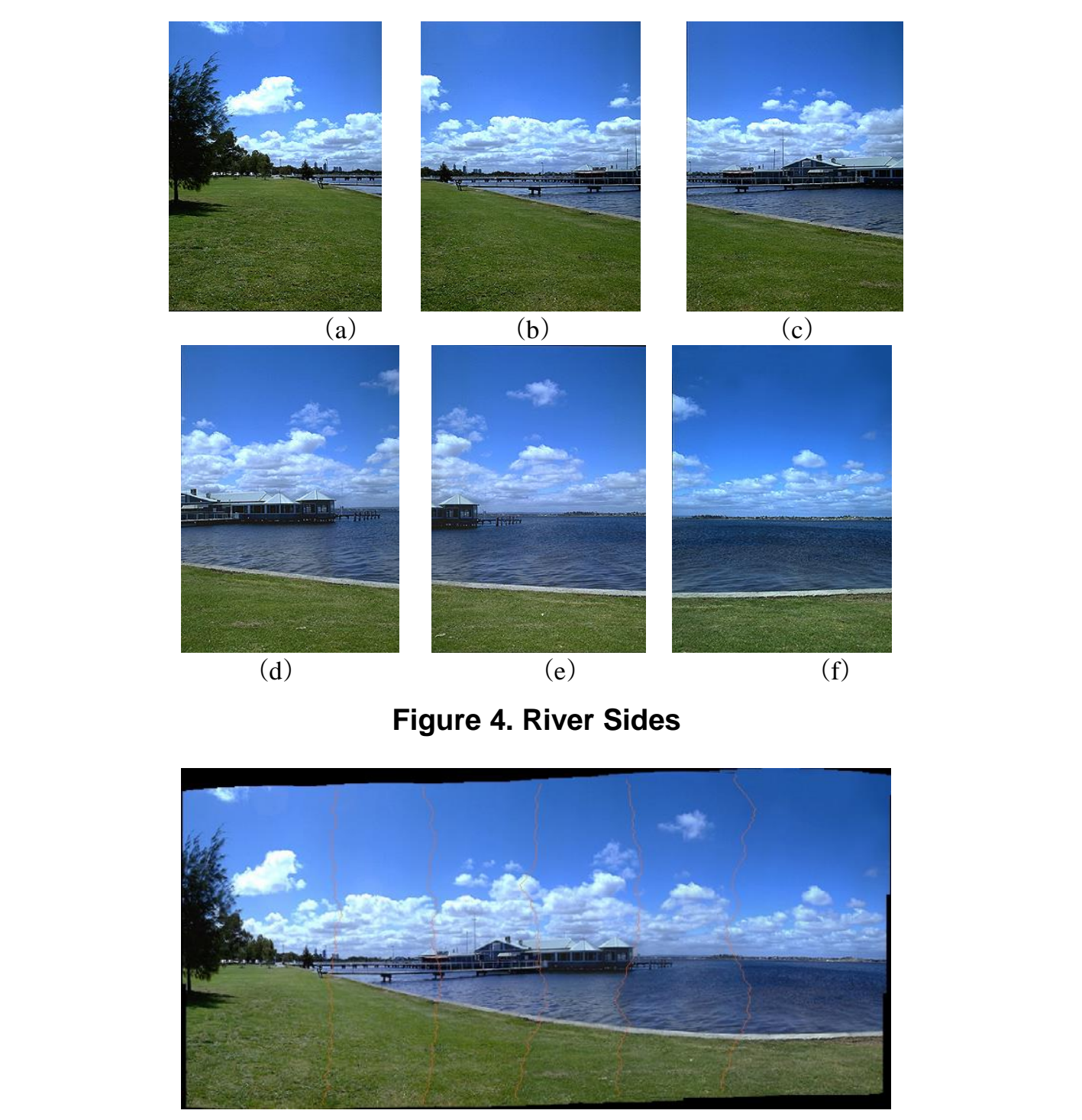

Figure 5. The Optimal Stitching Line Searching and Stitching

Searching the optimal stitching line in the overlap region of 6 source images, it can realize good mosaic effect. 


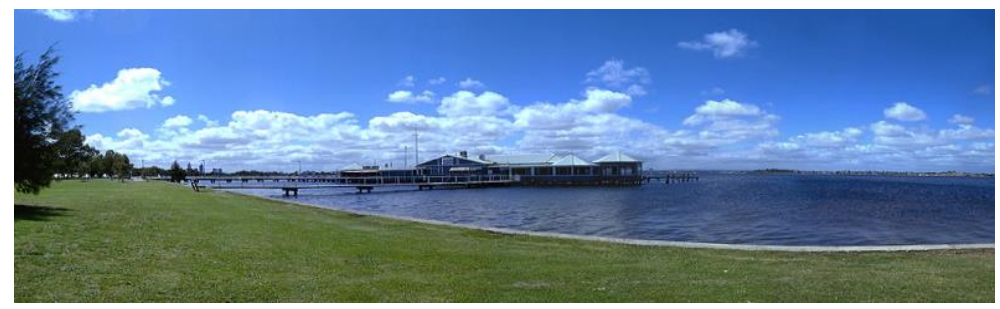

Figure 6. Image Fusion

From the Figure 6, Poisson image fusion technique can well realize changes accordingly that the background color of target image for color and illumination of the target pbject, and it realizes the ghost removal. The final stitching images have more real effect.

\section{Conclusions and Discussion}

This paper presents the detection method of overlap Tegion of stitching images, it uses the images optimal in the overlap region to obtain a optimal stitching line which the structure error is least, and the boundaries between two images, achieving the optimal stitching line search, improving the speed and precision of image stitching. And the Poisson image fusion algorithm to eliminate the ghost artifacts eaused by imagestitehing, in order to realize image fusion of the color difference images and yemove gbost artifacts due to moving objects, achieve brightness uniformity correction of stitching Images, and removes and repair ghost artifacts caused by moving object By experimental verification, the classic max flow / min cut method in the graph cut algorithm, can achieve a cost or segmentation of minimum energy, and obtain the optimal stitching line Røisson image fusion algorithm can realize the ghost artifacts removal, can bbtain high quality stitching images, and remove ghost artifacts in the process of moving, it has a good effect in the application. But the speed is still slow, still need to be further improved.

\section{Acknowledgements}

The work is supported by Hei Longiiang province Fund (F201339) Thanks Mr. Xie Wu for his invaluable comments of the work.

\section{References}

[1] Y. Boykov and O. Veksler, "Graph cuts in vision and graphics: theories and applications", Handbook of Mathematical Models in Computer Vision, New York: Springer, (2006).

[2] B. W. Cherkassky and A. V. Goldberg, "On implementing the push-relabel method for the maximum flow problem", Algorithmica, vol. 4, no. 19, (1997).

[3] A. V. Goldberg and R. E. Tarjan, "A new approach to the maximum flow problem", Journal of the ACM, vol. 4, no. 35, (1988).

[4] L. Ford and D. Fulkerson, "Flows in Networks", Princeton: Prince-ton University Press (1962).

[5] W. Jian, W. Shigang and C. Liwei, et al. "Adaptive Stereo Video Object Segmentation Based on Depth and Spatio-temporal Information. Proceedings of the Computer Science and Information Engineering", vol. 4, no. 7, (2009).

[6] Y. Boykov and G. Funka-Lea, "Graph cuts and efficient N-D image segmentation", International Journal of Computer Vision, vol. 2, no. 70, (2006).

[7] L. Songtao and Y. Fuliang, "The Basic Principle and its New Advances of Image Segmentation Methods Based on Graph Cut", ACTA Automatica Sinica, vol. 6, no. 38, (2012).

[8] R. Guo, "Automatic image stitching based on graph cut", Beijing (2012).

[9] L. Chunxiao, "Studies on Image Completion Based on the Large Displacement View Hangzhou (2009).

[10] P. P’erez, M. Gangnet, and A. Blake, "Poisson image editing”, ACM Trans. Graph, vol. 3, no. 22, (2003). 